\title{
Electricity Derivatives
}

\author{
Giovanni Barone-Adesi ${ }^{1} \quad$ Andrea Gigli ${ }^{2}$
}

This Draft: June, 3-th 2002

\begin{abstract}
In this paper we propose an algorithm for pricing derivatives written on electricity in an incomplete market setting. A discrete time model for price dynamics which embodies the main features of electricity price revealed by simple time series analysis is considered. We use jointly Binomial and Monte Carlo methods for pricing under a risk-neutral measure of which we prove the existence.
\end{abstract}

\footnotetext{
${ }^{1}$ E-mail address: Giovanni.Barone-Adesi@lu.unisi.ch

${ }^{2}$ E-mail address: $\underline{\text { Andrea.Gigli@lu.unisi.ch }}$
} 


\section{Introduction}

Electricity markets are becoming a popular field of research amongst academics because of the lack of appropriate models for describing electricity price behavior and pricing derivatives instruments. Models for price dynamics must take into account seasonalities and spiky behavior of jumps which seem hard to model by standard jump processes. Without good models for electricity price dynamics it is difficult to think about good models for futures, forward, swap or option pricing. As a result, very often pratictioners are forced to employ models based on the cost-of-carry for their needs.

There are several reasons which discourage the use of cost-of-carry models (see H. Geman and O. Vasiceck, 2001 and H. Geman and A. Roncoroni, 2001). Basically the most important arguments against them are related to the physical and temporal constraints of electricity as a non-storable commodity. Non-storability implies that arbitrage arguments cannot be used in defining a pricing model when electricity is the underlying of a derivative contract. Secondly, electricity transportation is based on the availability of line connections which can be damaged by rare and extreme events. Probably this kind of risk should be included in a good pricing model.

In this paper we do not try to solve all of the above problems but we simply propose an algorithm for pricing derivative instruments in an incomplete market setting. Our main idea is to include those features of electricity price behavior revealed by simple time series analysis in a discrete time model for electricity price dynamics. We use jointly Binomial and Monte Carlo methods for pricing under a risk-neutral measure. The existence of such a measure is discussed in the appendix.

The paper is organized as follows. In the next section we show the intuitions which are on the basis of our model, in section III we describe in detail how to implement the pricing and in section IV we present some simulation results for American call options. The last section remarks our main findings and some still open issues.

\section{A discrete time model}

In the following, the electricity price at time point $t>0$ is denoted by $E_{t}$ and spikes are assumed to occur at random times and with random durations as percentual changes on the previous price level. A spike is defined as a positive random percentual change in the previous price level of electricity and it is denoted as $S_{t}$ when it happens at time $t$. The function $D\left(S_{\tau}\right): \mathbb{R}^{+} \rightarrow \mathbb{N}^{+}$ returns the duration of the most recent spike occurred at time $\tau<t$. We also define $Q\left(S_{\tau}\right): \mathbb{R}^{+} \rightarrow \mathbb{N}^{+}$as the time at which the spike will break down. The diffusion is $X_{t}$, its parameters are $\mu$ and $\sigma$ and depends on $\varepsilon_{t} \sim N(0,1)$. Electricity prices are assumed to follow

$$
\Delta E_{t}=\Delta f\left(X_{t}, t\right)+W_{t} S_{t} E_{t-1}-Z_{t} S_{\tau} E_{t-1}, \quad E_{0}=X_{0}
$$

where

$$
\begin{gathered}
\Delta X_{t}=\mu X_{t} \Delta t+\sigma X_{t} \sqrt{\Delta t} \varepsilon_{t} \\
W_{t}=\left\{\begin{array}{l}
1 \text { with probability } q_{t} \\
0 \text { with probability } 1-q_{t}
\end{array}\right.
\end{gathered}
$$



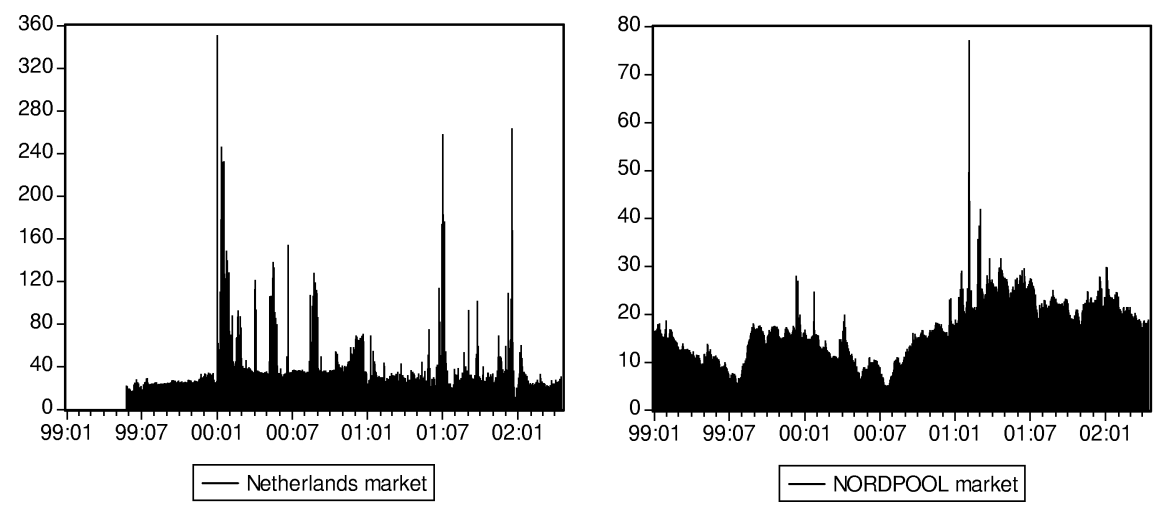

Figure 1: Electricity spot prices from Netherlands and NordPool markets.

$$
Z_{t}=\left\{\begin{array}{l}
1 \text { if } t=Q\left(S_{\tau}\right) \\
0 \text { otherwise }
\end{array}\right.
$$

The function $f\left(X_{t}, t\right)$ may allow for seasonalities in the diffusion, otherwise we may choose $f\left(X_{t}, t\right)=X_{t}$. The probability $q_{t}$ is the probability that a spike is realized at time $t$, provided $t>Q\left(S_{\tau}\right)$. The duration of spike occurred at time $t^{(i)}, D\left(S_{t^{(i)}}\right)$, is assumed to be independent of spike magnitude and price level.

Equation (1) defines changes in electricity prices in an additive way. The first component represents a diffusion, the second allows for positive percentual changes when a new spike happens and the third one models the collapsing of electricity prices when a spike breaks down. The last two components affect prices only when the indicator functions $W_{t}$ or $Z_{t}$ are equal to 1 . The former depends on the probability that a spike happens, which we assume to be zero if the previous spike is still affecting prices, the latter is equal to one only at the time the previous spike breaks down.

For modeling purposes it is useful to define $p_{t}=\lambda(t) \Delta t$ as the unconditional probability of spike arrivals in $\Delta t$ and to carry out the simulations using this measure. Indeed, we may simply simulate spikes in $t>Q\left(S_{\tau}\right)$ using $p_{t}$. Another advantage of using $p_{t}$ to simulate spike arrivals is that it is easier to include seasonal dependence and dependence from previous spike realizations in the spike frequency parameter.

For example, $m$ multiple peridiodicities may be included defining the spike frequency parameter like $\lambda(t)=\sum_{i=1}^{m} \omega_{i} \sin \left(2 \pi \gamma_{i} t^{i}\right)$, where time $t$ is expressed as a fraction of the year and $\omega_{i}$ and $\gamma_{i}$ are parameters specified to match periodicities. Alternatively, dependence on the previous $n$ realizations of the pure spike process can be introduced defining $\lambda(t)=\bar{\lambda}+\sum_{k=1}^{n} \theta^{t} \lambda(t-k) \mathbb{I}(t-k)$, where $0<\theta<1$ and $\mathbb{I}(j-k)$ is an indicator function which takes value 1 if a spike has happened at time $t-k$, and zero otherwise.

Another possible extension of our formulation regards the way the electricity price reverts to the diffusion process. If we call $t^{(i)}$ the time of the $i$-th spike, it 
would be not difficult to model the decline of the electricity price over the period $D\left(S_{t^{(i)}}\right)$ on a smoothed fashion rather than considering an istantaneous adjustment toward the diffusion process after $Q\left(S_{t^{(i)}}\right)$. Anyway, in the remaining of this paper we consider the simplest case, with $p_{t}=\lambda \Delta t$.

\section{Derivative Pricing}

In this section we show how it is possible to implement an algorithm that is consistent with the model described in the previous section. The pricing of contracts such as Forwards or Swaps is not very complicate given that we can express the price in $T$ of the former under a risk-adjusted measure as

$F_{T}=\mathbb{E}\left[E_{(N-1) \Delta t}+\Delta f\left(X_{N \Delta t}, N \Delta t\right)+W_{N \Delta t} S_{N \Delta t} E_{(N-1) \Delta t}-Z_{N \Delta t} S_{\tau} E_{(N-1) \Delta t}\right]$

where $T=N \Delta t$. The price of the latter in 0 is

$$
S W_{0}=\sum_{i=1}^{M}\left(F_{T_{i}}-L\right) e^{-r_{i} T_{i}}
$$

where $r_{i}$ is the discount rate for maturity $T_{i}$ and $L$ is the fixed price observed at the inception of the swap. The current fixed price for maturity $M$ is determined solving $S W_{0}=0$.

The pricing of options is not immediate and in the remaining of this section we will show how to implement our model for them. We define options as rights to either buy or sell a given quantity of energy at the current spot price.

On the basis of the previous considerations and for the sake of clearness we list below the specific assumptions used during the implementation.

- The distribution of the spike sizes follows a lognormal distribution. We assume that the spiky behavior in electricity prices is governed by a random positive percentage variation in the level of the diffusion process with mode $S_{M}$ and variance 0.04 .

- Spikes happen at random times but they cannot cumulate. We generate spikes as realizations of a random processes driven by $p_{t}$ 's but we put some constraints to get a simulation under $q_{t}$ 's. For example, considering a binomial tree with period $\Delta t$, number of levels $N=T / \Delta t$, we may use the condition that draws from the uniform distribution over the interval $(0,1)$ be smaller than $\lambda \Delta t$ to simulate $N$ realizations of the variable $\mathbb{I}_{t}$, taking value 1 if a spike happens and zero otherwise. Here $\lambda$ serves as spike frequency, that is the expected number of spikes in a year under $p_{t}$ 's. In practice, simulation under $q_{t}$ 's is possible imposing that a nonzero realization of $\mathbb{I}_{t}$ be considered as a realization of a spike only if it happens after the previous spike is broken down.

- Spike duration is independent of spike frequency. We use an exponential random variable with parameter $\theta$ to draw spike durations, $D\left(S_{t}\right)$. We set time in terms of years, i.e. $\theta=0.01$ implies an expected duration of the spike is about 3 days.

The pricing is based on a three-step procedure. Let $\Delta t=T / N$, where $N$ is the number of intervals in which we discretize the time to maturity $T$ : 


\begin{tabular}{|llll|cccc|}
\hline & & & & \multicolumn{3}{|c|}{$S_{M}=0.1$} & \multicolumn{2}{c|}{$S_{M}=0.5$} \\
\cline { 5 - 8 } & & & & $K=120$ & $K=100$ & $K=120$ & $K=100$ \\
\hline$T=0.5$ & $\theta=0.01$ & $\lambda=1$ & $\sigma=0.20$ & 01.1375 & 07.0594 & 1.9645 & 07.3608 \\
$T=0.5$ & $\theta=0.01$ & $\lambda=1$ & $\sigma=0.35$ & 04.3546 & 11.2197 & 04.7711 & 11.4557 \\
$T=0.5$ & $\theta=0.01$ & $\lambda=3$ & $\sigma=0.20$ & 01.3416 & 07.3741 & 02.3670 & 08.1194 \\
$T=0.5$ & $\theta=0.01$ & $\lambda=3$ & $\sigma=0.35$ & 04.5267 & 11.6207 & 05.4795 & 12.8859 \\
$T=0.5$ & $\theta=0.03$ & $\lambda=1$ & $\sigma=0.20$ & 01.2208 & 07.4976 & 02.0696 & 08.8299 \\
$T=0.5$ & $\theta=0.03$ & $\lambda=1$ & $\sigma=0.35$ & 04.6356 & 11.5720 & 05.4623 & 12.6670 \\
$T=0.5$ & $\theta=0.03$ & $\lambda=3$ & $\sigma=0.20$ & 01.6977 & 08.1432 & 04.9431 & 11.9138 \\
$T=0.5$ & $\theta=0.03$ & $\lambda=3$ & $\sigma=0.35$ & 04.9571 & 12.0864 & 07.9667 & 15.0492 \\
$T=1.0$ & $\theta=0.01$ & $\lambda=1$ & $\sigma=0.20$ & 03.3493 & 10.5975 & 03.7466 & 11.2411 \\
$T=1.0$ & $\theta=0.01$ & $\lambda=1$ & $\sigma=0.35$ & 09.0318 & 16.2801 & 09.6239 & 17.0490 \\
$T=1.0$ & $\theta=0.01$ & $\lambda=3$ & $\sigma=0.20$ & 03.6694 & 11.1631 & 05.2833 & 13.1754 \\
$T=1.0$ & $\theta=0.01$ & $\lambda=3$ & $\sigma=0.35$ & 09.3584 & 16.7175 & 10.6889 & 17.9083 \\
$T=1.0$ & $\theta=0.03$ & $\lambda=1$ & $\sigma=0.20$ & 03.6148 & 11.0441 & 05.0901 & 12.4233 \\
$T=1.0$ & $\theta=0.03$ & $\lambda=1$ & $\sigma=0.35$ & 09.2296 & 16.5490 & 10.6972 & 17.9176 \\
$T=1.0$ & $\theta=0.03$ & $\lambda=3$ & $\sigma=0.20$ & 04.0952 & 11.6818 & 07.8177 & 14.4743 \\
$T=1.0$ & $\theta=0.03$ & $\lambda=3$ & $\sigma=0.35$ & 09.6601 & 17.4878 & 12.6447 & 20.9894 \\
\hline
\end{tabular}

Table 1: European call option prices obtained by the algorithm using 1,000 realizations of a spike process for some values of $T, \theta, \lambda, \sigma$. The mode of the distribution of spike intensities is $S_{M}$ and $N=100$.

1. In the first step we build a tree based on the diffusion process with parameter $\mu$ and $\sigma$;

2. In the second we simulate 1,000 pathways. Each pathway includes $N$ spike realizations along the tree according to

$$
I_{t}=\left\{\begin{array}{l}
1 \text { if } u_{t}<\lambda \times \Delta t \\
0 \text { otherwise }
\end{array}\right.
$$

where $u_{t} \sim U(0,1)$. For each time interval in which a spike arrives we draw the percentage change in $X_{t}, S_{t}$, from a lognormal distribution with mode $S_{M}$ and variance 0.04 . At the same time the duration of each spike occurrence is drawn from an exponential distribution with parameter $\theta$. The process with spikes is obtained multiplying the nodes of the tree from $t^{(i)}$ to $T\left(S_{t^{(i)}}\right)$ by $1+S_{t^{(i)}}$ for the $i$-th spike, being careful to avoid multiple spikes in the same periods. An example is shown in figure (2).

3. The last step requires the computation of the option price along each of the 1,000 trees. We then take their mean as a way to integrate over the different spike process realizations.

European options prices are influenced by the spike effect only if a spike arrives or is not yet broken down in the last period. The prices on each tree can be computed by the binomial method as the discounted option payoffs weighted for the risk neutral probabilities, given the strike price $K$. The average price across trees is the option price.

The price of an American option cannot be determined exactly but we can define the upper and lower bounds for the fair price. The lower bound is computed using an approximate condition for early option exercise. In fact it can 


\begin{tabular}{|ccc|ccc|}
\hline & & & $K=80$ & $K=100$ & $K=120$ \\
\hline$T=1.0$ & $\sigma=0.20$ & $N=100$ & 20.6834 & 7.6466 & 2.0647 \\
$T=1.0$ & $\sigma=0.20$ & $N=500$ & 20.6797 & 7.6594 & 2.0546 \\
$T=1.0$ & $\sigma=0.35$ & $N=100$ & 24.1465 & 13.3365 & 7.0071 \\
$T=1.0$ & $\sigma=0.35$ & $N=500$ & 24.1413 & 13.3588 & 6.9810 \\
$T=0.5$ & $\sigma=0.20$ & $N=100$ & 20.1149 & 5.5126 & 0.7068 \\
$T=0.5$ & $\sigma=0.20$ & $N=500$ & 20.1168 & 5.5224 & 0.7046 \\
$T=0.5$ & $\sigma=0.35$ & $N=100$ & 21.8756 & 9.6306 & 3.5556 \\
$T=0.5$ & $\sigma=0.35$ & $N=500$ & 21.8630 & 9.6478 & 3.5445 \\
\hline
\end{tabular}

Table 2: American call option prices obtained by standard binomial pricing for some values of $T, \sigma, N$ and $K$. The starting value of the underlying price is 100 and it is assumed to follow a simple diffusion.

be shown that if

$$
E_{t} e^{-\sigma \sqrt{\Delta t}(N \Delta t-t)}-K>0
$$

then it is rational to exercise the American call option in $t$, provided that no other spike happens later. The upper bound can be found going backward along each tree and averaging over the option prices obtained for each realization of the spike process.

\section{Simulation results}

Following the model just described we compute American call option price bounds assuming that the price of Electricity is $100 \$$ at time 0 and the risk free rate is $5 \%$. In Table (2) we report American option prices under the usual Black-Scholes diffusion model for comparison. In Tables (4) - (15) we have computed derivative prices considering different values for the time to maturity (1 year and 6 months), strike $(80,100,120)$, volatility (20\% and $35 \%)$, spike magnitude (lognormal mode equal to $10 \%$ and $50 \%$ ), frequency (1 and 3 expected number of spikes each year) and duration ( 0.01 and 0.03 years). The last two columns of the tables shows the call price computed using the sufficient condition, $\underline{C}_{0}$, and the backward recursion on the binomial tree, $\bar{C}_{0}$.

The number of periods in which we have divided the time to maturity is not really important for pricing. In practice what really matter about the choice of time steps is to avoid that several spikes with duration lower then $\Delta t$ be excluded while valuation is carried out. In a more general setting, we may set $\Delta t$ for each replication only after having determined the shortest duration of spikes for a given replication.

At first look, prices obtained under our model are much higher then those reported in Table (2). The difference is due to the inclusion of the spike process and the differences are particularly sensible to the degree of moneyness, time to maturity, $\lambda$ and $S_{M}$. The diffusion coefficient $\sigma$ has little importance because the risk it represents is overwhelmed by those implied by the spiky behavior of the electricity prices.

Considering the prices obtained including the condition in Equation (2) it appears that these are close to $\overline{C_{0}}$ when we refer to in-the-money options and the spread increases as long as moneyness decreases, reaching the highest level 


\begin{tabular}{crrr}
\hline & & $t-$ Statistic & $P$-values \\
\hline$\hat{\beta}_{1}$ & 0.016370 & 0.004893 & 0.00111 \\
$\hat{\beta}_{2}$ & 0.023631 & 0.000529 & $2.96 E-45$ \\
$\hat{\beta}_{3}$ & -0.018936 & 0.003423 & $5.65 E-13$ \\
$R^{2}$ & & & \\
$F-$ statistic & 1022.514 & & 0.000000 \\
AIC & -5.780151 & & \\
\hline
\end{tabular}

Table 3: Estimation result from model (3).

when we are pricing out-of-the-money options, spikes are big and relatively frequent $(\lambda=3)$. In these cases $\underline{C}_{0}$ is however significantly greater than the corresponding European value in Table (2) because of the possibility of spikes occurring before the last period. That is different from the classical result in financial markets, that out-of-the money American options have values close to corresponding European options.

In the tables reporting pricing results there is evidence that the strategy implied in Equation (2) sometimes leads to a significant loss of value on early exercise. Therefore Equation (2) is not always adequate for pricing options and we need to correct the price in these cases. The spread is small when $T, \lambda$ and $S_{M}$ are small. In order to find a relation between $\bar{C}_{0}-\underline{C}_{0}$ and the price process determinants, we express the spread standardized by the strike price as a function of the spike intensity, the product of the frequency parameter and time to maturity and the moneyness degree. The simple linear model

$$
\left(\frac{\bar{C}_{0}-\underline{C}_{0}}{K}\right)_{i}=\beta_{1} S_{M, i}+\beta_{2} T_{i} \lambda_{i}+\beta_{3}\left(\frac{E_{0}}{K}\right)_{i}+u_{i}
$$

is estimated for pricing settings with $S_{M} \geq 0.5$ and $N=100$. Results in Table (3) show that this simple specification explains well the difference between the bounds and it has the feature to be quite general to avoid overfitting. The relation between upper and lower boundary is explained by Equation (4).

$$
\bar{C}_{0}=\underline{C}_{0}+K\left(\hat{\beta}_{1} S_{M}+\hat{\beta}_{2} T \lambda+\hat{\beta}_{3} \frac{E_{0}}{K}\right)
$$

The largest spreads occur when spikes are very frequent $(\lambda=5)$. The complete simulation approach should be used in these cases having a new simulation begin from each node where early exercise is potentially convenient. Detailed results are shown in Tables (4) - (15).

\section{Concluding Remarks}

We present a model for the dynamics of electricity spot prices and we describe some applications of the model for pricing derivative instruments. The application proposed in detail is the pricing of American call options, assuming a 
constant spike frequency, lognormally distributed spike magnitude and exponentially distributed spike durations. For this kind of derivative an exact fair price cannot be determined simply. It possible to find the upper and lower bound of the fair price under a risk-adjusted measure. The model can be generalized considering time dependent parameters and include seasonalities and even multiple periodicities as we have exemplified for $\lambda$.

Simulations results have shown that prices obtained under our algorithm are very different from those obtained by standard option pricing models. The lower bound is very easy to compute. The upper bound requires a greater effort. Often the two bounds are fairly close, suggesting that the lower bound is a close approximation of the fair price under most circumstances. However the bounds differ for out-of-the money options with high spike frequencies.

It is clear from the results that the most relevant determinants of the option price level are the parameters of the spike process. It would be useful to enrich the model in this direction, adding seasonalities and stylized facts to this component. Moreover, our pricing models have been computed under the risk-neutral measure. The existence of this measure is discussed in the appendix but the relationship between the physical and the risk-neutral measures is an important topic for future research. We believe that spike risk is largely idiosyncratic and this justifies the binomial pricing method as advocated by Merton (Merton R., 1992), but only long series of market data may cast light on this issue.

\section{Appendix}

Let $F$ be the forward price for delivery at $T$, where $T$ is any relevant time. Define $F^{*}$ as the forward price of a contract that delivers electricity if no spike is in progress at $T$. If a spike is in progress $F^{*}$ delivers against an additional payment equal to the spike intensity ${ }^{1}$. Let $M$ be the expected intensity of the spike at $T$. Define

$$
\pi=\frac{\left(F-F^{*}\right)}{M}
$$

then a risk-neutral measure exists if and only if $0<\pi<1$.

PROOF: From the definition of $\pi$.

$$
F=\pi M+(1-\pi) 0+\int X d \widetilde{P}
$$

where $X$ is the spot price at time $T$ and $\widetilde{P}$ is the forward risk neutral measure for the diffusion process without spikes. A similar proof holds for the risk neutral measure defined by futures contracts.

\section{Bibliography}

\section{References}

[1] H. Geman and O. Vasiceck (2001) Forward and future contracts on non storable commodities: the case of electricity, Preprint.

\footnotetext{
${ }^{1}$ Interruptible contracts, traded in the United States, have prices similar to $F^{*}$.
} 
[2] H. Geman and A. Roncoroni (2001) A class of marked point processes for modeling electricity prices, ESSEC working paper.

[3] L. Julio and E. Schwartz (2002) Electricity prices and power derivatives: evidence from the Nordic Power Exchange, Review of Derivatives Research $5,5-50$.

[4] R. Merton (1992), Continuous time finance, Blackwell, Cambridge, USA. 


\begin{tabular}{|ccccc|cc|}
\hline ITM & $S_{M}=0.1$ & & & & $\underline{C}_{0}$ & $\bar{C}_{0}$ \\
\hline$T=0.5$ & $\theta=0.01$ & $\lambda=1$ & $\sigma=0.20$ & $N=100$ & 29.8750 & 29.9848 \\
$T=0.5$ & $\theta=0.01$ & $\lambda=1$ & $\sigma=0.20$ & $N=500$ & 29.5986 & 29.7658 \\
$T=0.5$ & $\theta=0.01$ & $\lambda=1$ & $\sigma=0.35$ & $N=100$ & 30.5422 & 30.7317 \\
$T=0.5$ & $\theta=0.01$ & $\lambda=1$ & $\sigma=0.35$ & $N=500$ & 30.1514 & 30.3610 \\
$T=0.5$ & $\theta=0.01$ & $\lambda=3$ & $\sigma=0.20$ & $N=100$ & 37.6916 & 38.3850 \\
$T=0.5$ & $\theta=0.01$ & $\lambda=3$ & $\sigma=0.20$ & $N=500$ & 36.9241 & 38.0500 \\
$T=0.5$ & $\theta=0.01$ & $\lambda=3$ & $\sigma=0.35$ & $N=100$ & 37.8888 & 38.9504 \\
$T=0.5$ & $\theta=0.01$ & $\lambda=3$ & $\sigma=0.35$ & $N=500$ & 37.7231 & 38.9613 \\
$T=0.5$ & $\theta=0.03$ & $\lambda=1$ & $\sigma=0.20$ & $N=100$ & 29.5485 & 29.6705 \\
$T=0.5$ & $\theta=0.03$ & $\lambda=1$ & $\sigma=0.20$ & $N=500$ & 29.9932 & 30.1945 \\
$T=0.5$ & $\theta=0.03$ & $\lambda=1$ & $\sigma=0.35$ & $N=100$ & 30.5383 & 30.6589 \\
$T=0.5$ & $\theta=0.03$ & $\lambda=1$ & $\sigma=0.35$ & $N=500$ & 30.2876 & 30.5165 \\
$T=0.5$ & $\theta=0.03$ & $\lambda=3$ & $\sigma=0.20$ & $N=100$ & 37.7446 & 38.3995 \\
$T=0.5$ & $\theta=0.03$ & $\lambda=3$ & $\sigma=0.20$ & $N=500$ & 37.1316 & 38.1575 \\
$T=0.5$ & $\theta=0.03$ & $\lambda=3$ & $\sigma=0.35$ & $N=100$ & 37.8791 & 38.8375 \\
$T=0.5$ & $\theta=0.03$ & $\lambda=3$ & $\sigma=0.35$ & $N=500$ & 37.0842 & 38.2996 \\
\hline
\end{tabular}

Table 4: American call option prices obtained by the algorithm using 1,000 realizations of a spike process for some values of $T, \theta, \lambda, \sigma, N$. The mode of the distribution of spike intensities is $S_{M}$ and the strike is 80 .

\begin{tabular}{|ccccc|cc|}
\hline ITM & $S_{M}=0.1$ & & & & $\underline{C}_{0}$ & $\bar{C}_{0}$ \\
\hline$T=1.0$ & $\theta=0.01$ & $\lambda=1$ & $\sigma=0.20$ & $N=100$ & 36.1214 & 36.7029 \\
$T=1.0$ & $\theta=0.01$ & $\lambda=1$ & $\sigma=0.20$ & $N=500$ & 36.2104 & 36.8911 \\
$T=1.0$ & $\theta=0.01$ & $\lambda=1$ & $\sigma=0.35$ & $N=100$ & 37.2837 & 38.1680 \\
$T=1.0$ & $\theta=0.01$ & $\lambda=1$ & $\sigma=0.35$ & $N=500$ & 37.2801 & 38.2733 \\
$T=1.0$ & $\theta=0.01$ & $\lambda=3$ & $\sigma=0.20$ & $N=100$ & 42.3448 & 44.6130 \\
$T=1.0$ & $\theta=0.01$ & $\lambda=3$ & $\sigma=0.20$ & $N=500$ & 41.4840 & 44.7876 \\
$T=1.0$ & $\theta=0.01$ & $\lambda=3$ & $\sigma=0.35$ & $N=100$ & 42.1189 & 45.3512 \\
$T=1.0$ & $\theta=0.01$ & $\lambda=3$ & $\sigma=0.35$ & $N=500$ & 41.3902 & 45.5511 \\
$T=1.0$ & $\theta=0.03$ & $\lambda=1$ & $\sigma=0.20$ & $N=100$ & 36.0188 & 36.5551 \\
$T=1.0$ & $\theta=0.03$ & $\lambda=1$ & $\sigma=0.20$ & $N=500$ & 35.3979 & 36.1548 \\
$T=1.0$ & $\theta=0.03$ & $\lambda=1$ & $\sigma=0.35$ & $N=100$ & 36.8613 & 37.7634 \\
$T=1.0$ & $\theta=0.03$ & $\lambda=1$ & $\sigma=0.35$ & $N=500$ & 37.2322 & 38.2289 \\
$T=1.0$ & $\theta=0.03$ & $\lambda=3$ & $\sigma=0.20$ & $N=100$ & 42.1325 & 44.5772 \\
$T=1.0$ & $\theta=0.03$ & $\lambda=3$ & $\sigma=0.20$ & $N=500$ & 41.3921 & 44.4825 \\
$T=1.0$ & $\theta=0.03$ & $\lambda=3$ & $\sigma=0.35$ & $N=100$ & 42.0098 & 45.2732 \\
$T=1.0$ & $\theta=0.03$ & $\lambda=3$ & $\sigma=0.35$ & $N=500$ & 41.0580 & 45.2355 \\
\hline
\end{tabular}

Table 5: American call option prices obtained by the algorithm using 1,000 realizations of a spike process for some values of $T, \theta, \lambda, \sigma, N$. The mode of the distribution of spike intensities is $S_{M}$ and the strike is 80 . 


\begin{tabular}{|ccccc|cc|}
\hline ITM & $S_{M}=0.5$ & & & & $\underline{C}_{0}$ & $\bar{C}_{0}$ \\
\hline$T=0.5$ & $\theta=0.01$ & $\lambda=1$ & $\sigma=0.20$ & $N=100$ & 46.3913 & 46.4801 \\
$T=0.5$ & $\theta=0.01$ & $\lambda=1$ & $\sigma=0.20$ & $N=500$ & 46.5027 & 46.9213 \\
$T=0.5$ & $\theta=0.01$ & $\lambda=1$ & $\sigma=0.35$ & $N=100$ & 46.8861 & 47.2384 \\
$T=0.5$ & $\theta=0.01$ & $\lambda=1$ & $\sigma=0.35$ & $N=500$ & 47.3166 & 47.9577 \\
$T=0.5$ & $\theta=0.01$ & $\lambda=3$ & $\sigma=0.20$ & $N=100$ & 71.5896 & 72.7866 \\
$T=0.5$ & $\theta=0.01$ & $\lambda=3$ & $\sigma=0.20$ & $N=500$ & 71.0383 & 73.5544 \\
$T=0.5$ & $\theta=0.01$ & $\lambda=3$ & $\sigma=0.35$ & $N=100$ & 71.3235 & 73.7031 \\
$T=0.5$ & $\theta=0.01$ & $\lambda=3$ & $\sigma=0.35$ & $N=500$ & 70.5442 & 73.9954 \\
$T=0.5$ & $\theta=0.03$ & $\lambda=1$ & $\sigma=0.20$ & $N=100$ & 45.7253 & 45.9190 \\
$T=0.5$ & $\theta=0.03$ & $\lambda=1$ & $\sigma=0.20$ & $N=500$ & 46.6874 & 47.0690 \\
$T=0.5$ & $\theta=0.03$ & $\lambda=1$ & $\sigma=0.35$ & $N=100$ & 47.2720 & 47.5610 \\
$T=0.5$ & $\theta=0.03$ & $\lambda=1$ & $\sigma=0.35$ & $N=500$ & 46.6034 & 47.2365 \\
$T=0.5$ & $\theta=0.03$ & $\lambda=3$ & $\sigma=0.20$ & $N=100$ & 73.7413 & 74.6968 \\
$T=0.5$ & $\theta=0.03$ & $\lambda=3$ & $\sigma=0.20$ & $N=500$ & 71.0943 & 73.3377 \\
$T=0.5$ & $\theta=0.03$ & $\lambda=3$ & $\sigma=0.35$ & $N=100$ & 71.5541 & 73.1945 \\
$T=0.5$ & $\theta=0.03$ & $\lambda=3$ & $\sigma=0.35$ & $N=500$ & 70.1634 & 73.0487 \\
\hline
\end{tabular}

Table 6: American call option prices obtained by the algorithm using 1,000 realizations of a spike process for some values of $T, \theta, \lambda, \sigma, N$. The mode of the distribution of spike intensities is $S_{M}$ and the strike is 80 .

\begin{tabular}{|ccccc|cc|}
\hline ITM & $S_{M}=0.5$ & & & $\underline{C}_{0}$ & $\bar{C}_{0}$ \\
\hline$T=1.0$ & $\theta=0.01$ & $\lambda=1$ & $\sigma=0.20$ & $N=100$ & 62.5343 & 63.5429 \\
$T=1.0$ & $\theta=0.01$ & $\lambda=1$ & $\sigma=0.20$ & $N=500$ & 62.1316 & 63.5358 \\
$T=1.0$ & $\theta=0.01$ & $\lambda=1$ & $\sigma=0.35$ & $N=100$ & 62.2228 & 63.8457 \\
$T=1.0$ & $\theta=0.01$ & $\lambda=1$ & $\sigma=0.35$ & $N=500$ & 63.7876 & 65.6090 \\
$T=1.0$ & $\theta=0.01$ & $\lambda=3$ & $\sigma=0.20$ & $N=100$ & 84.9457 & 88.7413 \\
$T=1.0$ & $\theta=0.01$ & $\lambda=3$ & $\sigma=0.20$ & $N=500$ & 82.4418 & 89.0597 \\
$T=1.0$ & $\theta=0.01$ & $\lambda=3$ & $\sigma=0.35$ & $N=100$ & 83.5325 & 89.2921 \\
$T=1.0$ & $\theta=0.01$ & $\lambda=3$ & $\sigma=0.35$ & $N=500$ & 81.9741 & 89.6744 \\
$T=1.0$ & $\theta=0.03$ & $\lambda=1$ & $\sigma=0.20$ & $N=100$ & 64.3828 & 65.4066 \\
$T=1.0$ & $\theta=0.03$ & $\lambda=1$ & $\sigma=0.20$ & $N=500$ & 61.3676 & 63.4218 \\
$T=1.0$ & $\theta=0.03$ & $\lambda=1$ & $\sigma=0.35$ & $N=100$ & 62.4802 & 63.8345 \\
$T=1.0$ & $\theta=0.03$ & $\lambda=1$ & $\sigma=0.35$ & $N=500$ & 63.2104 & 65.3867 \\
$T=1.0$ & $\theta=0.03$ & $\lambda=3$ & $\sigma=0.20$ & $N=100$ & 85.6443 & 89.3603 \\
$T=1.0$ & $\theta=0.03$ & $\lambda=3$ & $\sigma=0.20$ & $N=500$ & 83.3663 & 89.9129 \\
$T=1.0$ & $\theta=0.03$ & $\lambda=3$ & $\sigma=0.35$ & $N=100$ & 82.9634 & 88.9521 \\
$T=1.0$ & $\theta=0.03$ & $\lambda=3$ & $\sigma=0.35$ & $N=500$ & 80.6769 & 89.0712 \\
\hline
\end{tabular}

Table 7: American call option prices obtained by the algorithm using 1,000 realizations of a spike process for some values of $T, \theta, \lambda, \sigma, N$. The mode of the distribution of spike intensities is $S_{M}$ and the strike is 80 . 


\begin{tabular}{|ccccc|cc|}
\hline ATM & $S_{M}=0.1$ & & & & $\underline{C}_{0}$ & $\bar{C}_{0}$ \\
\hline$T=0.5$ & $\theta=0.01$ & $\lambda=1$ & $\sigma=0.20$ & $N=100$ & 12.9826 & 13.2223 \\
$T=0.5$ & $\theta=0.01$ & $\lambda=1$ & $\sigma=0.20$ & $N=500$ & 13.0809 & 13.2710 \\
$T=0.5$ & $\theta=0.01$ & $\lambda=1$ & $\sigma=0.35$ & $N=100$ & 15.9707 & 16.2493 \\
$T=0.5$ & $\theta=0.01$ & $\lambda=1$ & $\sigma=0.35$ & $N=500$ & 15.5470 & 15.9175 \\
$T=0.5$ & $\theta=0.01$ & $\lambda=3$ & $\sigma=0.20$ & $N=100$ & 18.6140 & 19.9391 \\
$T=0.5$ & $\theta=0.01$ & $\lambda=3$ & $\sigma=0.20$ & $N=500$ & 18.3560 & 19.7200 \\
$T=0.5$ & $\theta=0.01$ & $\lambda=3$ & $\sigma=0.35$ & $N=100$ & 20.1706 & 21.9318 \\
$T=0.5$ & $\theta=0.01$ & $\lambda=3$ & $\sigma=0.35$ & $N=500$ & 19.8078 & 21.4986 \\
$T=0.5$ & $\theta=0.03$ & $\lambda=1$ & $\sigma=0.20$ & $N=100$ & 12.7450 & 12.9948 \\
$T=0.5$ & $\theta=0.03$ & $\lambda=1$ & $\sigma=0.20$ & $N=500$ & 13.0198 & 13.2713 \\
$T=0.5$ & $\theta=0.03$ & $\lambda=1$ & $\sigma=0.35$ & $N=100$ & 15.5891 & 15.9733 \\
$T=0.5$ & $\theta=0.03$ & $\lambda=1$ & $\sigma=0.35$ & $N=500$ & 15.9066 & 16.3703 \\
$T=0.5$ & $\theta=0.03$ & $\lambda=3$ & $\sigma=0.20$ & $N=100$ & 18.5443 & 19.7896 \\
$T=0.5$ & $\theta=0.03$ & $\lambda=3$ & $\sigma=0.20$ & $N=500$ & 18.1380 & 19.4866 \\
$T=0.5$ & $\theta=0.03$ & $\lambda=3$ & $\sigma=0.35$ & $N=100$ & 19.9624 & 21.7101 \\
$T=0.5$ & $\theta=0.03$ & $\lambda=3$ & $\sigma=0.35$ & $N=500$ & 20.0721 & 21.9510 \\
\hline
\end{tabular}

Table 8: American call option prices obtained by the algorithm using 1,000 realizations of a spike process for some values of $T, \theta, \lambda, \sigma, N$. The mode of the distribution of spike intensities is $S_{M}$ and the strike is 100 .

\begin{tabular}{|ccccc|cc|}
\hline ATM & $S_{M}=0.1$ & & & & $\underline{C}_{0}$ & $\bar{C}_{0}$ \\
\hline$T=1.0$ & $\theta=0.01$ & $\lambda=1$ & $\sigma=0.20$ & $N=100$ & 18.5186 & 19.3222 \\
$T=1.0$ & $\theta=0.01$ & $\lambda=1$ & $\sigma=0.20$ & $N=500$ & 18.5474 & 19.4738 \\
$T=1.0$ & $\theta=0.01$ & $\lambda=1$ & $\sigma=0.35$ & $N=100$ & 22.0031 & 23.5042 \\
$T=1.0$ & $\theta=0.01$ & $\lambda=1$ & $\sigma=0.35$ & $N=500$ & 21.6968 & 23.3463 \\
$T=1.0$ & $\theta=0.01$ & $\lambda=3$ & $\sigma=0.20$ & $N=100$ & 21.8986 & 25.8139 \\
$T=1.0$ & $\theta=0.01$ & $\lambda=3$ & $\sigma=0.20$ & $N=500$ & 21.6284 & 25.9550 \\
$T=1.0$ & $\theta=0.01$ & $\lambda=3$ & $\sigma=0.35$ & $N=100$ & 23.4219 & 28.8784 \\
$T=1.0$ & $\theta=0.01$ & $\lambda=3$ & $\sigma=0.35$ & $N=500$ & 23.4479 & 29.0103 \\
$T=1.0$ & $\theta=0.03$ & $\lambda=1$ & $\sigma=0.20$ & $N=100$ & 18.6384 & 19.4981 \\
$T=1.0$ & $\theta=0.03$ & $\lambda=1$ & $\sigma=0.20$ & $N=500$ & 18.6182 & 19.7847 \\
$T=1.0$ & $\theta=0.03$ & $\lambda=1$ & $\sigma=0.35$ & $N=100$ & 21.9422 & 23.4627 \\
$T=1.0$ & $\theta=0.03$ & $\lambda=1$ & $\sigma=0.35$ & $N=500$ & 21.4499 & 23.0300 \\
$T=1.0$ & $\theta=0.03$ & $\lambda=3$ & $\sigma=0.20$ & $N=100$ & 22.2485 & 26.2281 \\
$T=1.0$ & $\theta=0.03$ & $\lambda=3$ & $\sigma=0.20$ & $N=500$ & 21.8104 & 25.7352 \\
$T=1.0$ & $\theta=0.03$ & $\lambda=3$ & $\sigma=0.35$ & $N=100$ & 23.7080 & 29.1479 \\
$T=1.0$ & $\theta=0.03$ & $\lambda=3$ & $\sigma=0.35$ & $N=500$ & 23.0125 & 28.8168 \\
\hline
\end{tabular}

Table 9: American call option prices obtained by the algorithm using 1,000 realizations of a spike process for some values of $T, \theta, \lambda, \sigma, N$. The mode of the distribution of spike intensities is $S_{M}$ and the strike is 100 . 


\begin{tabular}{|ccccc|cc|}
\hline ATM & $S_{M}=0.5$ & & & & $\underline{C}_{0}$ & $\bar{C}_{0}$ \\
\hline$T=0.5$ & $\theta=0.01$ & $\lambda=1$ & $\sigma=0.20$ & $N=100$ & 29.2419 & 29.4422 \\
$T=0.5$ & $\theta=0.01$ & $\lambda=1$ & $\sigma=0.20$ & $N=500$ & 29.3349 & 29.8570 \\
$T=0.5$ & $\theta=0.01$ & $\lambda=1$ & $\sigma=0.35$ & $N=100$ & 31.2238 & 31.7673 \\
$T=0.5$ & $\theta=0.01$ & $\lambda=1$ & $\sigma=0.35$ & $N=500$ & 31.7474 & 32.4010 \\
$T=0.5$ & $\theta=0.01$ & $\lambda=3$ & $\sigma=0.20$ & $N=100$ & 52.0969 & 54.0428 \\
$T=0.5$ & $\theta=0.01$ & $\lambda=3$ & $\sigma=0.20$ & $N=500$ & 51.5843 & 54.7923 \\
$T=0.5$ & $\theta=0.01$ & $\lambda=3$ & $\sigma=0.35$ & $N=100$ & 52.5536 & 55.5433 \\
$T=0.5$ & $\theta=0.01$ & $\lambda=3$ & $\sigma=0.35$ & $N=500$ & 52.0156 & 55.8333 \\
$T=0.5$ & $\theta=0.03$ & $\lambda=1$ & $\sigma=0.20$ & $N=100$ & 28.6794 & 28.9403 \\
$T=0.5$ & $\theta=0.03$ & $\lambda=1$ & $\sigma=0.20$ & $N=500$ & 29.5670 & 29.9929 \\
$T=0.5$ & $\theta=0.03$ & $\lambda=1$ & $\sigma=0.35$ & $N=100$ & 31.5247 & 32.0603 \\
$T=0.5$ & $\theta=0.03$ & $\lambda=1$ & $\sigma=0.35$ & $N=500$ & 31.1372 & 31.8324 \\
$T=0.5$ & $\theta=0.03$ & $\lambda=3$ & $\sigma=0.20$ & $N=100$ & 54.1376 & 55.8021 \\
$T=0.5$ & $\theta=0.03$ & $\lambda=3$ & $\sigma=0.20$ & $N=500$ & 51.8601 & 54.5755 \\
$T=0.5$ & $\theta=0.03$ & $\lambda=3$ & $\sigma=0.35$ & $N=100$ & 52.8258 & 55.0459 \\
$T=0.5$ & $\theta=0.03$ & $\lambda=3$ & $\sigma=0.35$ & $N=500$ & 51.5920 & 54.9025 \\
\hline
\end{tabular}

Table 10: American call option prices obtained by the algorithm using 1,000 realizations of a spike process for some values of $T, \theta, \lambda, \sigma, N$. The mode of the distribution of spike intensities is $S_{M}$ and the strike is 100 .

\begin{tabular}{|ccccc|cc|}
\hline ATM & $S_{M}=0.5$ & & & & $\underline{C}_{0}$ & $\bar{C}_{0}$ \\
\hline$T=1.0$ & $\theta=0.01$ & $\lambda=1$ & $\sigma=0.20$ & $N=100$ & 44.6127 & 46.0056 \\
$T=1.0$ & $\theta=0.01$ & $\lambda=1$ & $\sigma=0.20$ & $N=500$ & 44.3551 & 46.0665 \\
$T=1.0$ & $\theta=0.01$ & $\lambda=1$ & $\sigma=0.35$ & $N=100$ & 45.8577 & 47.6927 \\
$T=1.0$ & $\theta=0.01$ & $\lambda=1$ & $\sigma=0.35$ & $N=500$ & 47.0810 & 49.1886 \\
$T=1.0$ & $\theta=0.01$ & $\lambda=3$ & $\sigma=0.20$ & $N=100$ & 64.4447 & 69.5363 \\
$T=1.0$ & $\theta=0.01$ & $\lambda=3$ & $\sigma=0.20$ & $N=500$ & 62.1149 & 69.8241 \\
$T=1.0$ & $\theta=0.01$ & $\lambda=3$ & $\sigma=0.35$ & $N=100$ & 63.4817 & 70.4774 \\
$T=1.0$ & $\theta=0.01$ & $\lambda=3$ & $\sigma=0.35$ & $N=500$ & 62.4178 & 70.8811 \\
$T=1.0$ & $\theta=0.03$ & $\lambda=1$ & $\sigma=0.20$ & $N=100$ & 46.3017 & 47.7776 \\
$T=1.0$ & $\theta=0.03$ & $\lambda=1$ & $\sigma=0.20$ & $N=500$ & 43.6654 & 45.9829 \\
$T=1.0$ & $\theta=0.03$ & $\lambda=1$ & $\sigma=0.35$ & $N=100$ & 45.9468 & 47.6148 \\
$T=1.0$ & $\theta=0.03$ & $\lambda=1$ & $\sigma=0.35$ & $N=500$ & 46.6011 & 48.9510 \\
$T=1.0$ & $\theta=0.03$ & $\lambda=3$ & $\sigma=0.20$ & $N=100$ & 64.3964 & 70.1050 \\
$T=1.0$ & $\theta=0.03$ & $\lambda=3$ & $\sigma=0.20$ & $N=500$ & 62.9806 & 70.6535 \\
$T=1.0$ & $\theta=0.03$ & $\lambda=3$ & $\sigma=0.35$ & $N=100$ & 62.7815 & 70.2011 \\
$T=1.0$ & $\theta=0.03$ & $\lambda=3$ & $\sigma=0.35$ & $N=500$ & 61.0449 & 70.3456 \\
\hline
\end{tabular}

Table 11: American call option prices obtained by the algorithm using 1,000 realizations of a spike process for some values of $T, \theta, \lambda, \sigma, N$. The mode of the distribution of spike intensities is $S_{M}$ and the strike is 100 . 


\begin{tabular}{|ccccc|cc|}
\hline OTM & $S_{M}=0.1$ & & & & $\underline{C}_{0}$ & $\bar{C}_{0}$ \\
\hline$T=0.5$ & $\theta=0.01$ & $\lambda=1$ & $\sigma=0.20$ & $N=100$ & 02.7645 & 03.1451 \\
$T=0.5$ & $\theta=0.01$ & $\lambda=1$ & $\sigma=0.20$ & $N=500$ & 02.7800 & 03.1604 \\
$T=0.5$ & $\theta=0.01$ & $\lambda=1$ & $\sigma=0.35$ & $N=100$ & 06.0904 & 06.6743 \\
$T=0.5$ & $\theta=0.01$ & $\lambda=1$ & $\sigma=0.35$ & $N=500$ & 05.9745 & 06.6759 \\
$T=0.5$ & $\theta=0.01$ & $\lambda=3$ & $\sigma=0.20$ & $N=100$ & 04.1711 & 05.9766 \\
$T=0.5$ & $\theta=0.01$ & $\lambda=3$ & $\sigma=0.20$ & $N=500$ & 04.2016 & 06.0476 \\
$T=0.5$ & $\theta=0.01$ & $\lambda=3$ & $\sigma=0.35$ & $N=100$ & 07.0232 & 09.7962 \\
$T=0.5$ & $\theta=0.01$ & $\lambda=3$ & $\sigma=0.35$ & $N=500$ & 07.1634 & 09.8647 \\
$T=0.5$ & $\theta=0.03$ & $\lambda=1$ & $\sigma=0.20$ & $N=100$ & 02.8829 & 03.2678 \\
$T=0.5$ & $\theta=0.03$ & $\lambda=1$ & $\sigma=0.20$ & $N=500$ & 02.8327 & 03.2921 \\
$T=0.5$ & $\theta=0.03$ & $\lambda=1$ & $\sigma=0.35$ & $N=100$ & 06.0658 & 06.8635 \\
$T=0.5$ & $\theta=0.03$ & $\lambda=1$ & $\sigma=0.35$ & $N=500$ & 06.2296 & 06.9138 \\
$T=0.5$ & $\theta=0.03$ & $\lambda=3$ & $\sigma=0.20$ & $N=100$ & 04.2663 & 06.1963 \\
$T=0.5$ & $\theta=0.03$ & $\lambda=3$ & $\sigma=0.20$ & $N=500$ & 04.2507 & 06.2362 \\
$T=0.5$ & $\theta=0.03$ & $\lambda=3$ & $\sigma=0.35$ & $N=100$ & 07.2157 & 09.9773 \\
$T=0.5$ & $\theta=0.03$ & $\lambda=3$ & $\sigma=0.35$ & $N=500$ & 06.9814 & 09.8383 \\
\hline
\end{tabular}

Table 12: American call option prices obtained by the algorithm using 1,000 realizations of a spike process for some values of $T, \theta, \lambda, \sigma, N$. The mode of the distribution of spike intensities is $S_{M}$ and the strike is 120 .

\begin{tabular}{|ccccc|cc|}
\hline OTM & $S_{M}=0.1$ & & & & $\underline{C}_{0}$ & $\bar{C}_{0}$ \\
\hline$T=1.0$ & $\theta=0.01$ & $\lambda=1$ & $\sigma=0.20$ & $N=100$ & 05.8406 & 07.3510 \\
$T=1.0$ & $\theta=0.01$ & $\lambda=1$ & $\sigma=0.20$ & $N=500$ & 06.1324 & 07.5509 \\
$T=1.0$ & $\theta=0.01$ & $\lambda=1$ & $\sigma=0.35$ & $N=100$ & 10.6793 & 13.0845 \\
$T=1.0$ & $\theta=0.01$ & $\lambda=1$ & $\sigma=0.35$ & $N=500$ & 10.7492 & 13.0215 \\
$T=1.0$ & $\theta=0.01$ & $\lambda=3$ & $\sigma=0.20$ & $N=100$ & 05.9835 & 11.3237 \\
$T=1.0$ & $\theta=0.01$ & $\lambda=3$ & $\sigma=0.20$ & $N=500$ & 05.9308 & 11.0995 \\
$T=1.0$ & $\theta=0.01$ & $\lambda=3$ & $\sigma=0.35$ & $N=100$ & 09.4740 & 17.2252 \\
$T=1.0$ & $\theta=0.01$ & $\lambda=3$ & $\sigma=0.35$ & $N=500$ & 09.3099 & 17.0246 \\
$T=1.0$ & $\theta=0.03$ & $\lambda=1$ & $\sigma=0.20$ & $N=100$ & 06.1037 & 07.6047 \\
$T=1.0$ & $\theta=0.03$ & $\lambda=1$ & $\sigma=0.20$ & $N=500$ & 05.7246 & 07.4503 \\
$T=1.0$ & $\theta=0.03$ & $\lambda=1$ & $\sigma=0.35$ & $N=100$ & 10.7727 & 13.1104 \\
$T=1.0$ & $\theta=0.03$ & $\lambda=1$ & $\sigma=0.35$ & $N=500$ & 10.5357 & 13.3294 \\
$T=1.0$ & $\theta=0.03$ & $\lambda=3$ & $\sigma=0.20$ & $N=100$ & 05.9233 & 11.3846 \\
$T=1.0$ & $\theta=0.03$ & $\lambda=3$ & $\sigma=0.20$ & $N=500$ & 05.9878 & 11.3433 \\
$T=1.0$ & $\theta=0.03$ & $\lambda=3$ & $\sigma=0.35$ & $N=100$ & 09.3918 & 17.3599 \\
$T=1.0$ & $\theta=0.03$ & $\lambda=3$ & $\sigma=0.35$ & $N=500$ & 09.1443 & 17.2594 \\
\hline
\end{tabular}

Table 13: American call option prices obtained by the algorithm using 1,000 realizations of a spike process for some values of $T, \theta, \lambda, \sigma, N$. The mode of the distribution of spike intensities is $S_{M}$ and the strike is 120 . 


\begin{tabular}{|ccccc|cc|}
\hline OTM & $S_{M}=0.5$ & & & & $\underline{C}_{0}$ & $\bar{C}_{0}$ \\
\hline$T=0.5$ & $\theta=0.01$ & $\lambda=1$ & $\sigma=0.20$ & $N=100$ & 17.6710 & 18.2194 \\
$T=0.5$ & $\theta=0.01$ & $\lambda=1$ & $\sigma=0.20$ & $N=500$ & 18.1478 & 18.7939 \\
$T=0.5$ & $\theta=0.01$ & $\lambda=1$ & $\sigma=0.35$ & $N=100$ & 19.7224 & 20.3172 \\
$T=0.5$ & $\theta=0.01$ & $\lambda=1$ & $\sigma=0.35$ & $N=500$ & 19.9204 & 20.7207 \\
$T=0.5$ & $\theta=0.01$ & $\lambda=3$ & $\sigma=0.20$ & $N=100$ & 34.6956 & 37.3718 \\
$T=0.5$ & $\theta=0.01$ & $\lambda=3$ & $\sigma=0.20$ & $N=500$ & 34.2901 & 38.0985 \\
$T=0.5$ & $\theta=0.01$ & $\lambda=3$ & $\sigma=0.35$ & $N=100$ & 35.2587 & 38.6438 \\
$T=0.5$ & $\theta=0.01$ & $\lambda=3$ & $\sigma=0.35$ & $N=500$ & 35.0963 & 38.8652 \\
$T=0.5$ & $\theta=0.03$ & $\lambda=1$ & $\sigma=0.20$ & $N=100$ & 17.8595 & 18.3396 \\
$T=0.5$ & $\theta=0.03$ & $\lambda=1$ & $\sigma=0.20$ & $N=500$ & 17.9709 & 18.4808 \\
$T=0.5$ & $\theta=0.03$ & $\lambda=1$ & $\sigma=0.35$ & $N=100$ & 19.8336 & 20.6625 \\
$T=0.5$ & $\theta=0.03$ & $\lambda=1$ & $\sigma=0.35$ & $N=500$ & 19.6689 & 20.4965 \\
$T=0.5$ & $\theta=0.03$ & $\lambda=3$ & $\sigma=0.20$ & $N=100$ & 35.1103 & 37.9307 \\
$T=0.5$ & $\theta=0.03$ & $\lambda=3$ & $\sigma=0.20$ & $N=500$ & 34.9616 & 38.3003 \\
$T=0.5$ & $\theta=0.03$ & $\lambda=3$ & $\sigma=0.35$ & $N=100$ & 35.0757 & 38.2893 \\
$T=0.5$ & $\theta=0.03$ & $\lambda=3$ & $\sigma=0.35$ & $N=500$ & 35.1366 & 38.8880 \\
\hline
\end{tabular}

Table 14: American call option prices obtained by the algorithm using 1,000 realizations of a spike process for some values of $T, \theta, \lambda, \sigma, N$. The mode of the distribution of spike intensities is $S_{M}$ and the strike is 120 .

\begin{tabular}{|ccccc|cc|}
\hline OTM & $S_{M}=0.5$ & & & & $\underline{C}_{0}$ & $\bar{C}_{0}$ \\
\hline$T=1.0$ & $\theta=0.01$ & $\lambda=1$ & $\sigma=0.20$ & $N=100$ & 29.7326 & 31.5437 \\
$T=1.0$ & $\theta=0.01$ & $\lambda=1$ & $\sigma=0.20$ & $N=500$ & 29.0364 & 31.1717 \\
$T=1.0$ & $\theta=0.01$ & $\lambda=1$ & $\sigma=0.35$ & $N=100$ & 32.2088 & 34.4994 \\
$T=1.0$ & $\theta=0.01$ & $\lambda=1$ & $\sigma=0.35$ & $N=500$ & 32.1511 & 34.3449 \\
$T=1.0$ & $\theta=0.01$ & $\lambda=3$ & $\sigma=0.20$ & $N=100$ & 44.4208 & 51.3083 \\
$T=1.0$ & $\theta=0.01$ & $\lambda=3$ & $\sigma=0.20$ & $N=500$ & 42.3072 & 51.2874 \\
$T=1.0$ & $\theta=0.01$ & $\lambda=3$ & $\sigma=0.35$ & $N=100$ & 43.8272 & 53.1472 \\
$T=1.0$ & $\theta=0.01$ & $\lambda=3$ & $\sigma=0.35$ & $N=500$ & 42.9827 & 52.8007 \\
$T=1.0$ & $\theta=0.03$ & $\lambda=1$ & $\sigma=0.20$ & $N=100$ & 30.5809 & 32.4505 \\
$T=1.0$ & $\theta=0.03$ & $\lambda=1$ & $\sigma=0.20$ & $N=500$ & 29.4335 & 31.3746 \\
$T=1.0$ & $\theta=0.03$ & $\lambda=1$ & $\sigma=0.35$ & $N=100$ & 32.1465 & 34.1942 \\
$T=1.0$ & $\theta=0.03$ & $\lambda=1$ & $\sigma=0.35$ & $N=500$ & 32.4565 & 35.1242 \\
$T=1.0$ & $\theta=0.03$ & $\lambda=3$ & $\sigma=0.20$ & $N=100$ & 43.6222 & 51.7772 \\
$T=1.0$ & $\theta=0.03$ & $\lambda=3$ & $\sigma=0.20$ & $N=500$ & 42.4879 & 51.3064 \\
$T=1.0$ & $\theta=0.03$ & $\lambda=3$ & $\sigma=0.35$ & $N=100$ & 43.2047 & 51.8437 \\
$T=1.0$ & $\theta=0.03$ & $\lambda=3$ & $\sigma=0.35$ & $N=500$ & 42.5801 & 52.9565 \\
\hline
\end{tabular}

Table 15: American call option prices obtained by the algorithm using 1,000 realizations of a spike process for some values of $T, \theta, \lambda, \sigma, N$. The mode of the distribution of spike intensities is $S_{M}$ and the strike is 120 . 

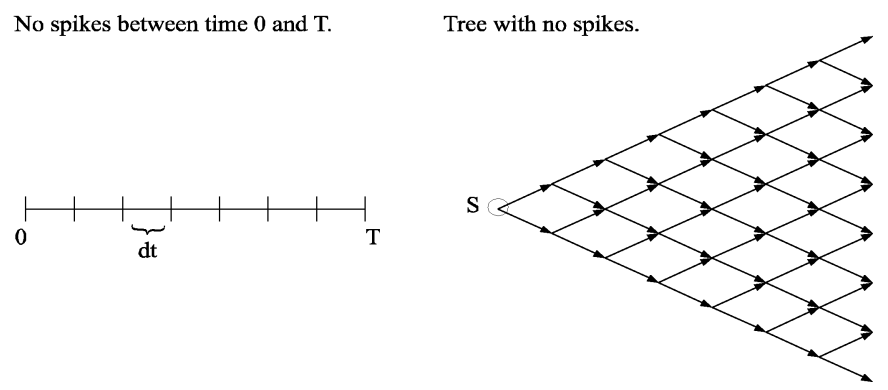

Spikes on period 1 and 5 .

Tree with spikes on period 1 and 5 .

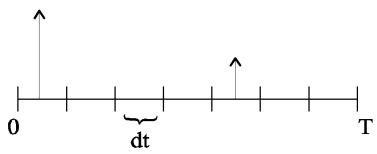

$\mathrm{s}$
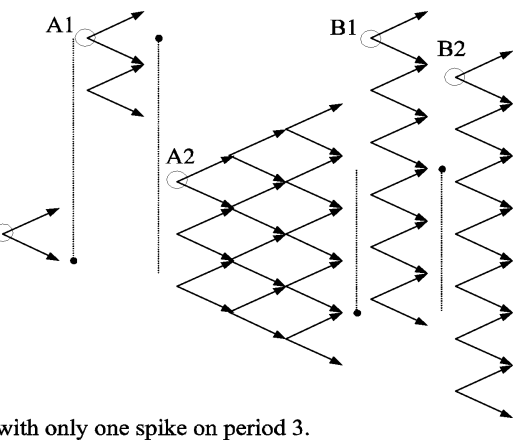

Spike on period 3.

Tree with only one spike on period 3.
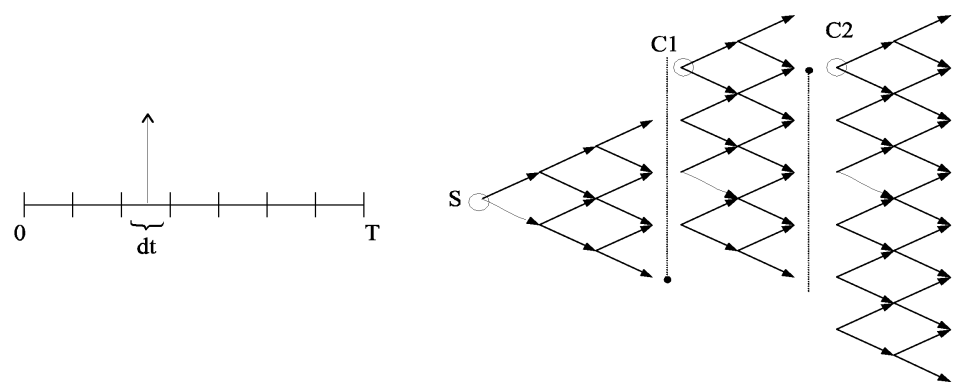

Figure 2: The figure show three examples of spike processes realizations over binomial trees. The number of levels affected by the spike is random, as well as the intensity of the spike magnitude. 